\title{
Higher Susceptibility to Sunburn is Associated with Decreased Plasma Glutamine and Increased Plasma Glutamate Levels among U.S. Women: An Analysis of the Nurses' Health Study I and II
}

Authors: Keming Yang, MD, $\mathrm{PhD}^{1}$, Xin Li, $\mathrm{ScD}^{2}$, Oana A Zeleznik, $\mathrm{PhD}^{3}$, A. Heather Eliassen, $\mathrm{ScD}^{3,4}$, Clary B. Clish, $\mathrm{PhD}^{5}$, Eunyoung Cho, $\mathrm{ScD}^{6,7}$, Ally-Khan B. Somani, MD, $\mathrm{PhD}^{8}, \mathrm{Abrar}$ A. Qureshi, MD, MPH ${ }^{6,7}$, Edward L. Giovannucci, MD, ScD ${ }^{1,3,4}$, Hongmei Nan, MD, $\mathrm{PhD}^{2,9}$

${ }^{1}$ Department of Nutrition, Harvard T.H. Chan School of Public Health, Boston, MA, 02115, USA.

${ }^{2}$ Department of Epidemiology, Richard M. Fairbanks School of Public Health, Indiana University, Indianapolis, IN 46202, USA.

${ }^{3}$ Channing Division of Network Medicine, Department of Medicine, Brigham and Women's Hospital and Harvard Medical School, Boston, MA, 02115, USA.

${ }^{4}$ Department of Epidemiology, Harvard T.H. Chan School of Public Health, Boston, MA, 02115, USA.

${ }^{5}$ Broad Institute of Massachusetts Institute of Technology and Harvard University, Cambridge, MA, 02142, USA.

${ }^{6}$ Department of Dermatology, Warren Alpert Medical School, Brown University, Providence, RI 02903, USA.

${ }^{7}$ Department of Epidemiology, School of Public Health, Brown University, Providence, RI 02903, USA.

${ }^{8}$ Department of Dermatology, Indiana University School of Medicine, Indianapolis, IN 46202, USA.

${ }^{9}$ Department of Global Health, Richard M. Fairbanks School of Public Health, Indiana University, Indianapolis, IN 46202, USA.

Correspondence to: Keming Yang, MD, PhD, Department of Nutrition, Harvard T.H. Chan School of Public Health, Boston, MA, 02115, USA. Email: kyang@ hsph.harvard.edu

Acknowledgements and Funding: This work is supported by NIH grants UM1 CA186107, R01 CA49449, U01 CA176726, R01 CA67262. The content is solely the responsibility of the authors and does not necessarily represent the official views of the National Institutes of Health. We would like to thank the participants and staff of the Nurses' Health Study (NHS) I and II for their valuable contributions. The authors assume full responsibility for analyses and interpretation of these data.

IRB approval status: The study protocol was approved by the Institutional Review Boards of the Brigham and Women's Hospital and the Harvard T.H. Chan School of Public Health.

Conflicts of Interest: None declared.

Manuscript word count: 500 out of 500

References: 5 of 5

Tables: 2 out of 2

Supplementary tables: 1 (available at doi:10.17632/h95pksd5jz.1)

This is the author's manuscript of the article published in final edited form as:

Yang, K., Li, X., Zeleznik, O. A., Eliassen, A. H., Clish, C. B., Cho, E., Somani, A.-K. B., Qureshi, A. A., Giovannucci, E. L., \& Nan, H. (2021). Higher susceptibility to sunburn is associated with decreased plasma glutamine and increased plasma glutamate levels among US women: An analysis of the Nurses' Health Study I and II. Journal of the American Academy of Dermatology. https://doi.org/10.1016/j.jaad.2020.12.081 
The metabolism of glutamine and glutamate, two important amino acids synthesized in the human body, may have an etiologic role in melanoma, an aggressive skin malignancy. ${ }^{1,2}$ Preclinical experiments and clinical trials have demonstrated that metabotropic glutamate receptor 1 blocker and glutamate-release inhibitor (e.g., Riluzole) can suppress melanoma cell migration, invasion, and proliferation. ${ }^{2}$ Additionally, inhibiting glutaminase, the enzyme that converts glutamine to glutamate, further reduced glutamate bioavailability and suppressed tumor progression. ${ }^{1}$ Susceptibility to sunburn, a pigmentary trait, is a well-known risk factor for melanoma. ${ }^{3}$ However, it is unclear whether plasma glutamate and glutamine are affected by this host factor even before cancer onset.

We hypothesized plasma glutamate/glutamine levels may differ by individuals' sunburn susceptibility. To test this hypothesis, we examined the association among 9,129 women from previous case-control studies nested within the Nurses' Health Study (NHS) I ( $n=5,981)$ and II $(n=3,148) .{ }^{4}$ Non-whites and participants with a history of skin cancer or any other malignancy prior to blood collection were excluded. Information on skin reaction after two or more hours' sun exposure as a child/adolescent was collected and grouped into four categories (i.e., none/some redness only, burn, painful burn, painful burn with blisters). Plasma glutamate and glutamine concentrations were obtained using liquid chromatography-tandem mass spectrometry (LC-MS) at the Broad Institute of MIT and Harvard University. Details of metabolite profiling methods have been published previously. ${ }^{5}$ Age and multivariable-adjusted linear regressions were performed and percentage differences in levels of glutamate, glutamine, and glutamate/glutamine ratio between sunburn susceptibility groups were calculated using the following equation: $[\exp (\beta$-coefficient $)-1] \times 100 \%$. All analyses were performed using SAS (Unix 9.4) 
Characteristics of participants by quintiles of glutamate/glutamine ratio are presented in

71 Table 1. We found a significant decreasing trend of glutamine [painful burn with blisters vs. reference: $-9.2 \%(-16.9 \%,-0.7 \%), P$-trend $=0.005]$, and significant increasing trends of glutamate [painful burn with blisters vs. reference: $19.9 \%(10.0 \%, 30.7 \%), P$-trend $<0.0001$ ] as well as glutamate/glutamine ratio [painful burn with blisters vs. reference: $22.8 \%(12.6 \%, 33.8 \%), P$ trend $<0.0001$ ] across sunburn susceptibility groups (Table 2). The association patterns were consistent and significant in the sensitivity analysis of controls-only samples [ $n=4,672$; painful burn with blisters vs. reference: $13.4 \%(0.6 \%, 27.8 \%)$ for glutamate, $-11.8 \%(-22.1 \%,-0.2 \%)$ for glutamine, $15.6 \%(2.6 \%, 30.2 \%)$ for glutamate/glutamine ratio]. We did not find significant interactions of sunburn susceptibility and selected covariates (i.e., age, smoking, body mass index, physical activity, alcohol consumption, Alternate Healthy Eating Index and personal history of diabetes, cardiovascular diseases or hypercholesterolemia in relation to plasma glutamate/glutamine ratio (all $P$-interaction $>0.05 / 7)$ (Table S1).

Overall, our study found that being susceptible to sunburn, especially painful burn with blisters, was associated with higher glutamate and lower glutamine in plasma among cancer-free participants. Although several limitations are acknowledged, such as the women-only and crosssectional study design, this is the first study demonstrating a potential association between sunburn susceptibility (a highly heritable trait and melanoma risk factor) and plasma glutamate/glutamine (a target of anti-melanoma therapy). Additionally, our epidemiologic findings provide evidence for future investigations in terms of whether glutamatergic signaling is a potential underlying pathway by which sunburn susceptibility alters melanoma risk. Further studies are needed to replicate our findings. 


\section{Supplementary File:}

Supplementary Table 1. Percentage differences (95\% CIs) in plasma glutamate/glutamine ratio according to sunburn susceptibility, stratified by selected covariates in all 9129 participants

References

1. Shah R, Singh SJ, Eddy K, Filipp FV, Chen S. Concurrent targeting of glutaminolysis and metabotropic glutamate receptor 1 (grm 1 ) reduces glutamate bioavailability in grm1(+) melanoma. Cancer research. 2019;79:1799-1809

2. Le MN, Chan JL, Rosenberg SA, Nabatian AS, Merrigan KT, Cohen-Solal KA, et al. The glutamate release inhibitor riluzole decreases migration, invasion, and proliferation of melanoma cells. The Journal of investigative dermatology. 2010;130:2240-2249

3. Wu S, Han J, Laden F, Qureshi AA. Long-term ultraviolet flux, other potential risk factors, and skin cancer risk: A cohort study. Cancer epidemiology, biomarkers \& prevention : a publication of the American Association for Cancer Research, cosponsored by the American Society of Preventive Oncology. 2014;23:1080-1089

4. Bao Y, Bertoia ML, Lenart EB, Stampfer MJ, Willett WC, Speizer FE, et al. Origin, methods, and evolution of the three nurses' health studies. American journal of public health. 2016;106:1573-1581

5. Mayers JR, Wu C, Clish CB, Kraft P, Torrence ME, Fiske BP, et al. Elevation of circulating branched-chain amino acids is an early event in human pancreatic adenocarcinoma development. Nat Med. 2014;20:1193-1198 
Table 1. Characteristics of 9,129 cancer-free participants from NHS I and II by quintiles of plasma glutamate/glutamine ratio ${ }^{1}$

\begin{tabular}{|c|c|c|c|c|c|}
\hline Characteristics & Quintile 1 & Quintile 2 & Quintile 3 & Quintile 4 & Quintile 5 \\
\hline Number of participants & 1825 & 1826 & 1826 & 1826 & 1826 \\
\hline Age at blood collection, years & $51.5(8.0)$ & $52.0(8.3)$ & $52.7(8.7)$ & $53.5(8.6)$ & $53.1(8.5)$ \\
\hline Plasma glutamate level, z-score & $-1.1(0.5)$ & $-0.5(0.5)$ & $-0.1(0.5)$ & $0.4(0.5)$ & $1.3(0.8)$ \\
\hline Plasma glutamine level, z-score & $0.5(0.9)$ & $0.2(0.9)$ & $0.1(0.9)$ & $-0.2(0.9)$ & $-0.6(1.0)$ \\
\hline Plasma glutamate/glutamine ratio, z-score & $-1.3(0.3)$ & $-0.6(0.1)$ & $-0.1(0.1)$ & $0.5(0.2)$ & $1.5(0.6)$ \\
\hline Painful burn or blistering after $\geq 2 \mathrm{~h}$ sun exposure, $\%$ & 13.5 & 15.1 & 18.2 & 20.2 & 21.3 \\
\hline Red or blonde hair, $\%^{2}$ & 14.8 & 16.1 & 15.6 & 17.8 & 17.7 \\
\hline$\geq 6$ moles on the extremity, $\%^{3}$ & 8.8 & 11.6 & 12.8 & 13.0 & 10.8 \\
\hline Average July noon-time erythemal $\mathrm{UV}, \mathrm{mW} / \mathrm{m} 2$ & $188.7(29.4)$ & $190.1(29.2)$ & $189.8(28.9)$ & $192.6(30.5)$ & $191.4(30.2)$ \\
\hline Body mass index, $\mathrm{kg} / \mathrm{m} 2$ & $23.7(3.5)$ & $24.6(4.2)$ & $25.8(4.7)$ & $26.8(5.3)$ & $27.8(6.1)$ \\
\hline Physical activity, metabolic equivalents, hours/wk & $17.1(18.8)$ & $17.5(25.7)$ & $15.7(19.1)$ & $17.0(25.8)$ & $15.2(18)$ \\
\hline \multicolumn{6}{|l|}{ Cigarette smoking } \\
\hline Never smoker, \% & 58.1 & 55.6 & 52.5 & 51.0 & 46.7 \\
\hline Past smoker, \% & 31.5 & 33.4 & 35.3 & 35.9 & 40.3 \\
\hline Current smoker, $\%$ & 10.4 & 10.9 & 12.2 & 13.1 & 13.0 \\
\hline Alcohol intake, $g / d$ & $4.6(7.8)$ & $5(9.0)$ & $5.2(9.3)$ & $5.6(10.2)$ & $5.2(9.4)$ \\
\hline Alternate healthy eating index & $46.6(10.0)$ & $46.4(10.0)$ & $46.4(9.6)$ & $46.3(9.9)$ & $46.5(9.7)$ \\
\hline History of cardiovascular diseases & 0.8 & 0.9 & 1.1 & 0.9 & 1.6 \\
\hline History of diabetes & 0.2 & 0.4 & 1.0 & 2.2 & 5.5 \\
\hline History of hypercholesterolemia & 30.0 & 34.5 & 32.8 & 37.7 & 37.8 \\
\hline
\end{tabular}

Note: ${ }^{1}$ Information presented was collected at the time of blood collection or at the questionnaire cycle closest to blood collection

133 (NHS I: 1989-1990, NHS II: 1996-1999) except for otherwise noted. Values are means (SD) for continuous variables, percentages for 134 categorical variables, and are standardized to the age distribution of the study population except for age.

$135{ }^{2}$ Natural color of hair at 21 and 18 was asked in NHS I and II, respectively.

$136{ }^{3}$ Total number of moles $>3 \mathrm{~mm}$ diameter was asked for the left arm in NHS I, for both lower legs in NHS II. 
Table 2. Percentage differences (95\% CIs) in plasma levels of glutamate, glutamine, and glutamate/glutamine ratio according to sunburn susceptibility among 9,129 cancer-free participants from NHS I and II

ring to sunburn susceptibility among 9,129 cancer-free participants from NHS I and III

\begin{tabular}{|c|c|c|c|c|c|}
\hline & $\begin{array}{c}\text { None or } \\
\text { some redness only }\end{array}$ & Burn & Painful burn & $\begin{array}{c}\text { Painful burn with } \\
\text { blisters }\end{array}$ & $\begin{array}{l}\text { P for } \\
\text { trend }\end{array}$ \\
\hline Number of participants & 5445 & 2042 & 1106 & 536 & \\
\hline \multicolumn{6}{|l|}{ Glutamate } \\
\hline Age-adjusted model & 0 (ref) & $4.1 \%(-1.1 \%, 9.5 \%)$ & $15.2 \%(8.0 \%, 22.9 \%)$ & $34.7 \%(23.3 \%, 47.1 \%)$ & $<0.0001$ \\
\hline Multivariable-adjusted model & 0 (ref) & $1.7 \%(-3.1 \%, 6.8 \%)$ & $7.0 \%(0.5 \%, 13.9 \%)$ & $19.9 \%(10.0 \%, 30.7 \%)$ & $<0.0001$ \\
\hline \multicolumn{6}{|l|}{ Glutamine } \\
\hline Age-adjusted model & 0 (ref) & $-0.3 \%(-5.2 \%, 4.8 \%)$ & $-8.4 \%(-14.1 \%,-2.4 \%)$ & $-10.1 \%(-17.7 \%,-1.9 \%)$ & 0.002 \\
\hline Multivariable-adjusted model & 0 (ref) & $-0.4 \%(-5.4 \%, 4.7 \%)$ & $-8.0 \%(-13.8 \%,-1.8 \%)$ & $-9.2 \%(-16.9 \%,-0.7 \%)$ & 0.005 \\
\hline \multicolumn{6}{|l|}{ Glutamate/glutamine ratio } \\
\hline Age-adjusted model & 0 (ref) & $4.3 \%(-0.8 \%, 9.7 \%)$ & $17.7 \%(10.4 \%, 25.6 \%)$ & $37.6 \%(26.0 \%, 50.3 \%)$ & $<0.0001$ \\
\hline Multivariable-adjusted model & 0 (ref) & $2.0 \%(-2.9 \%, 7.1 \%)$ & $9.6 \%(2.9 \%, 16.7 \%)$ & $22.8 \%(12.6 \%, 33.8 \%)$ & $<0.0001$ \\
\hline
\end{tabular}

42 Notes: Covariates adjusted in multivariable-adjusted model include continuous age, body mass index, physical activity, alcohol

143 consumption, alternate healthy eating index, smoking status (never, past, current), fasting status, case-control status in previous nested

144 case-control studies, history of diabetes, cardiovascular diseases or hypercholesterolemia, hair color (black/dark brown, light brown,

145 blonde, red), mole counts (none, 1-2, 3-5, 6+), and cohort. 
160

161

162

163

164

165

166
Supplementary Table 1. Percentage differences (95\% CIs) in plasma glutamate/glutamine ratio according to sunburn susceptibility, stratified by selected covariates among all 9,129 participants 